\title{
A Concepção das Mães sobre o Desenvolvimento da Comunicação Mãe-Bebêt ${ }^{1}$
}

\author{
Maria C. D. P. Lyra \\ Antonio Roazzi ${ }^{2}$ \\ Universidade Federal de Pernambuco
}

\begin{abstract}
RESUMO - Comparamos duas abordagens teórico-metodológicas distintas e investigamos a concepção que mães apresentam acerca do processo de desenvolvimento da comunicação mãe-bebê. Lyra e colaboradores propõem o modelo EEA, que integra uma sequiência de três padrões de organização do desenvolvimento da comunicação, denominados estabelecimento, extensão e abreviação (EEA). Esse modelo está baseado na observação longitudinal e na análise microgenética de estudos de caso a partir da perspectiva dos sistemas dinâmicos. Utilizando o procedimento de classificação múltipla, investigamos como esses três períodos do desenvolvimento da comunicação (EEA) são conceituados pelas mães. Foram pesquisadas 48 mães pertencentes a dois níveis sócio-econômicos. Os resultados encontrados fortalecem o modelo EEA, confirmando: a distinção entre as trocas face-a-face e aquelas mediadas pelo objeto (MOB); a diferenciação dos três períodos do desenvolvimento da comunicação (EEA) em ambas as trocas; e a distribuição ordenada (axial) desses três períodos, exceto, nesse último caso, para as trocas MOB, quando analisadas separadamente.
\end{abstract}

Palavras-chave: desenvolvimento da comunicação; mãe-bebê; concepção das mães; modelo EEA; teoria das facetas.

\section{Mother's Conceptualization of the Mother-Infant Communication Development}

\begin{abstract}
Two theoretical-methodological distinct perspectives are compared in order to study mother's conceptualization of the process of mother-infant development of communication. Based on dynamic systems perspective and relying on longitudinal microanalysis of case studies observations, Lyra and collaborators propose the EEA model for studying this process of development. This model integrates a sequence of three periods of development of this process, conceived as developmental patterns of organization: establishment, extension and abbreviation (EEA). Using a "multiple sorting procedure", we investigated mothers' conceptualization of these three periods of development. We examined 48 mothers, from two socioeconomic levels. The results support the EEA model confirming: the distinction between face-to-face and the exchanges mediated by the object (MOB); the differentiation among the three periods of communication development (EEA) in both type of exchanges; and, an order distribution (axial) of these three periods, except for the specific analysis of the MOB type of exchanges.
\end{abstract}

Key words: communication development; mother-infant; mother's conceptualization; EEA model; facet theory.

Este artigo tem um duplo objetivo. Comparar duas abordagens teórico-metodológicas distintas e investigar a concepção que mães apresentam acerca do desenvolvimento da comunicação mãe-bebê. Partindo da pesquisa desse processo de desenvolvimento investigado longitudinalmente e analisado de forma microgenética, focalizando a observação de estudos de casos, Lyra e colaboradores (Fogel \& Lyra, 1997; Lyra, 2000; Lyra, 2006a, Lyra, 2006b, Lyra, 2007, Lyra, no prelo; Lyra \& Souza, 2003; Lyra \& Winegar, 1997) propõem o modelo EEA para analisar e descrever esse desenvolvimento. Esse modelo, baseado na perspectiva dos Sistemas Dinâmicos, integra uma seqüência de três padrões de organização, denominados estabelecimento, extensão e abreviação (EEA), que descrevem períodos de quase-estabilidade do sistema de comunicação mãe-bebê durante os oito primeiros meses de vida pós-natal do bebê. Por outro lado, o Procedimento de Classificação Múltipla - PCM (Roazzi, 1995; Roazzi, Wilson \& Federicci, 2001), método de investigação utilizado no presente trabalho, nos oferece a possibilidade de explorar

\section{Financiamento CNPq.}

2 Endereço: Universidade Federal de Pernambuco, Pós-Graduação em Psicologia, $\mathrm{CFCH} 8^{\circ}$, andar, Cidade Universitária, Rua Acadêmico Hélio Ramos, s/n, Recife, PE, Brasil 50670-901.E-mail: marialyra2005@yahoo.com.br como esses períodos do desenvolvimento da comunicação são identificados e conceituados pelas mães.

Adotamos a perspectiva de que os relacionamentos interpessoais são sistemas de comunicação em desenvolvimento, sistemas que geram o significado para os participantes. Propomos que esse significado está diretamente relacionado à dinâmica das mudanças do sistema de comunicação, ou seja, ao seu desenvolvimento, desde o início da vida pós-natal do bebê. Considerando o desenvolvimento da comunicação mãe-bebê e o período aqui abordado, sugerimos que os períodos de maior estabilidade, que exibem padrões de organização característicos desse sistema de comunicação, produzem significados que podem ser detectados pelas mães ao conceituarem esse desenvolvimento.

\section{A Perspectiva dos Sistemas Dinâmicos e o Modelo EEA}

Sobretudo, a partir da última década do século passado, as pesquisas em Psicologia, particularmente Psicologia do Desenvolvimento, têm nos apresentado uma perspectiva teórica e metodológica especialmente voltada para a investigação do processo de mudança que ocorre em um tempo irreversível (Valsiner, 2002). Agrupada sob o rótulo de estudo dos sis- 
temas, concebidos como Sistemas Dinâmicos (Fogel, 1993; Fogel \& Lyra, 1997; Granott \& Parziale, 2002; Lewis, 2000; Thelen \& Smith, 1994; Thelen \& Ulrich, 1991; van Geert, 2002, 2003), essa perspectiva nos fornece uma série de conceitos que nos habilitam a investigar "como" o desenvolvimento ocorre, ou seja, o próprio processo de desenvolvimento.

Duas contribuições são especialmente dignas de nota: a) a possibilidade de incluir, na mesma estrutura conceitual, ambas as características do processo de desenvolvimento, ou seja, tanto os períodos de maior estabilidade como aqueles de mudança e b) a concepção de que o desenvolvimento do sistema se deve à capacidade de auto-organização inerente a todos os sistemas vivos, resultando na emergência de novos padrões de organização ao longo do tempo (Fogel, Lyra \& Valsiner, 1997; van Geert, 2003). Essa perspectiva tem sido utilizada para explorar diferentes aspectos do desenvolvimento, tais como o aspecto motor (Thelen \& Smith, 1994; Thelen \& Ulrich, 1991), o aspecto cognitivo (van Geert, 2002, 2003), a comunicação e a emoção (Fogel, 1993; Fogel \& Lyra, 1997; Lewis, 1995; Lyra, 2000, Lyra, 2006a, Lyra, 2006b, Lyra, 2007, Lyra, no prelo; Lyra \& Souza, 2003; Lyra \& Winegar, 1997; Pantoja \& Nelson-Goens, 2000; Pedrosa, Carvalho \& Império-Hamburger, 1997), a personalidade (Masterpasqua \& Perna, 1997) e o apego (Coleman \& Watson, 2000).

Considerando o desenvolvimento da comunicação no início da vida, Fogel (1993) e Lyra e colaboradores (Fogel \& Lyra, 1997; Lyra, 2000, Lyra, 2006a, Lyra, 2006b, Lyra, 2007, Lyra, no prelo; Lyra \& Souza, 2003; Lyra \& Winegar, 1997) têm contribuído com novas idéias conceituais e metodológicas que integram aspectos da perspectiva dos Sistemas Dinâmicos aplicados ao estudo da comunicação mãe-bebê no primeiro ano pós-natal de vida do bebê. Essas idéias achamse integradas no modelo EEA abaixo descrito.

\section{O Modelo EEA: Estabelecimento, Extensão e Abreviação}

O modelo EEA propõe que o processo de comunicação mãe-bebê seja construído obedecendo a uma seqüência de padrões organizacionais que descrevem períodos de quase-estabilidade do sistema de comunicação mãe-bebê. Essas trocas comunicativas, sem a mediação do objeto (face-a-face - FF) ou com essa mediação (mãe-objeto-bebê - MOB), exibem níveis desenvolvimentais que possibilitam incluir os aspectos generalizáveis e as particularidades presentes (Molenaar \& Valsiner, 2005) em cada díade mãe-bebê estudada. Ambos são inerentes à história de cada díade. Cada um desses padrões exibe um tipo característico de negociação entre os parceiros. Assim, esse conjunto de três conceitos visa a representar os processos subjacentes ao desenvolvimento da comunicação mãe-bebê, particularmente durante os primeiros oito meses de vida pós-natal do bebê.

Podemos definir o estabelecimento, a extensão e a abreviação da seguinte forma:

Estabelecimento. Por meio de sucessivas ou concomitantes ações dos parceiros, que "recortam" o fluxo de atividades da díade, pelo menos uma ação das trocas diádicas é construída de forma partilhada pela díade. Esta ação se torna "figura" em relação a um "fundo" de outras ações realizadas pela díade. Por exemplo, o olhar mútuo entre os parceiros, con- siderando as trocas FF, ou o olhar dos parceiros para o objeto, considerando as trocas MOB. São trocas de curta duração ${ }^{3}$.

Extensão. As ações previamente estabelecidas tornamse um "fundo" em relação ao qual a díade pode "recortar" e negociar, elaborando de modo mais prolongado, outras ações como "figuras". Por exemplo, nas trocas FF, tendo estabelecido o contato de olhar mútuo, podem ser negociados sorrisos, vocalizações, movimentos, etc., de forma elaborada e prolongada. Considerando o olhar conjunto dos parceiros para o objeto como fundo, os movimentos dos braços/mãos do bebê para o objeto podem ser negociados durante trocas prolongadas que focalizam esses movimentos.

Abreviação. Trocas de curta duração - inferior ou igual a seis turnos - executadas pela díade por um ajustamento mútuo rápido e fácil, em que as ações anteriormente negociadas e trabalhadas pela díade, de forma prolongada, aparecem de modo reduzido ou condensado (abreviados). Por exemplo, considerando as trocas $\mathrm{FF}$, a díade utiliza um rápido contato de olhar acompanhado ou não de algumas ações anteriormente negociadas (sorriso, movimentos, por exemplo). Dando outro exemplo, tanto a mãe como o bebê, de forma ajustada, rápida e suave, dirigem o olhar para um objeto, que é então oferecido pela mãe e imediatamente segurado pelo bebê.

\section{Estudo realizado}

Este trabalho pretende relacionar os conceitos de estabelecimento, extensão e abreviação acima definidos a outro tipo de estudo que busca identificar a concepção das mães sobre o desenvolvimento da comunicação. Ambos abordam os primeiros oito meses de vida pós-natal do bebê. Dessa maneira, comparam-se dois tipos de abordagens teóricometodológicas, sintetizadas da seguinte maneira: a) uma conceituação do desenvolvimento das trocas mãe-bebê como processo em constante transformação, investigado a partir da observação longitudinal e análise microgenética de estudos de caso (Fogel \& Lyra, 1997; Lyra, 2000, Lyra, 2006a, Lyra, 2006b, Lyra, 2007, Lyra, no prelo; Lyra \& Souza, 2003; Lyra \& Winegar, 1997) e b) outra, utilizando a Teoria das Facetas (Bilsky, 2003; Borg \& Shye, 1995; Canter, 1985; Guttman, 1991; Roazzi \& Dias, 2001; Silva, Lyra \& Roazzi, 2001), envolvendo uma estratégia de categorização e criação de sistemas de classificação para conceituar esse desenvolvimento. Investigamos, assim, a correspondência entre a descrição do processo de desenvolvimento da comunicação mãe-bebê, proposto pelo modelo EEA, e a maneira como as mães conceituam esse desenvolvimento. Trata-se, portanto, de relacionar uma abordagem voltada para o estudo do processo ou dinâmica do desenvolvimento com uma outra que investiga a capacidade de categorização das mães.

Os padrões de organização do sistema de comunicação abordados (EEA) foram identificados tanto para as trocas FF como para aquelas MOB, longitudinalmente vídeografadas (uma vez por semana) e microanaliticamente investigadas. A idéia de quase-estabilidade requer que concebamos o desenvolvimento como um processo em constante mudança. Portanto os conceitos de estabelecimento, extensão e abreviação

\footnotetext{
3 As referências a número de turnos como menor no estabelecimento e abreviação corresponde a inferior ou igual a seis turnos e maior na extensão, a partir de sete turnos.
} 
não capturam, exatamente, categorias estáveis, mas processos em constante transformação. De outra parte, a abordagem que fundamenta a investigação acerca da concepção das mães está baseada em categorizações razoavelmente estáveis das mães acerca desse desenvolvimento, envolvendo uma estratégia de criação de sistemas de classificação para conceituar esse desenvolvimento. Dessa forma, a importância deste estudo está na possibilidade de validar duas abordagens, tanto teórica como metodologicamente diversas.

Para verificar a conceituação das mães sobre esses três padrões de organização do desenvolvimento do sistema de comunicação mãe-bebê (EEA), foi investigada uma amostra de mães que não trabalhavam (metade habitando em um ambiente típico da classe operária e a outra metade habitando em ambiente característico da classe média), utilizando-se o Procedimento de Classificação Múltipla (PCM; e.g., Roazzi, 1995). Esse procedimento foi aplicado obedecendo a dois tipos de solicitação: a) tarefa de classificação livre, na qual as mães foram solicitadas a classificar livremente em grupos quaisquer 20 fotografias que exibiam momentos da comunicação mãe-bebê referentes aos padrões de organização EEA e b) tarefa de classificação guiada, na qual as mães foram solicitadas a ordenar, atribuindo um número a essas 20 fotografias. Metade das fotografias se referia às trocas FF e a outra metade às trocas MOB. Após cada classificação efetuada, solicitavam-se às mães que justificassem as suas escolhas, ou seja, as classificações efetuadas.

O Procedimento de Classificação Múltipla (PCM) é um dos procedimentos utilizados para explorar a maneira como as pessoas categorizam, criando sistemas de classificação. Trata-se de um procedimento aberto que possibilita aos participantes fornecer suas concepções exibidas nas classificações efetuadas acerca de um determinado assunto. Esse método foi desenvolvido a partir de procedimentos de auto-categorização de Sheriff e Sheriff (1969) e das atividades de classificação usadas por Vygotsky (1934/1962). Diferente de outros procedimentos de classificação, esse tipo de procedimento não requer nenhum conjunto pré-estabelecido na distribuição das fotos, deixando o investigado bastante livre para classificar o material exibido. A idéia subjacente é que quanto mais liberdade é dada à pessoa investigada, mais provavelmente o entrevistador irá apreender algo sobre ela e seu sistema de formação de conceitos. Nesse estudo, a classificação livre deixou o sujeito com inteira liberdade para classificar e, na classificação guiada, requeria-se apenas que as fotografias fossem ordenadas em sequiência, de acordo com o que as mães pensam que ocorreu entre ela e seu bebê ao longo do tempo. Exigia-se, assim, nessa última, uma ordenação histórico-temporal.

\section{Definindo aspectos da conceituação das mães sobre o desenvolvimento da comunicação mãe-bebê}

Dois aspectos ("facetas") da concepção de mães acerca do desenvolvimento da comunicação na primeira infância foram definidos: a) Trocas - caracterizadas em dois níveis: trocas FF e MOB; e b) Períodos - caracterizados em três níveis: estabelecimento, extensão e abreviação (EEA).

Nossa hipótese é que se os conceitos de EEA descrevem padrões de organização do desenvolvimento do sistema de comunicação mãe-bebê (tanto FF como MOB); então, esses conceitos podem estar presentes na conceituação das mães acerca da comunicação mãe-bebê no início da vida. Conseqüentemente, essa conceituação deve ser identificável ao se estudar as habilidades das mães na construção de um sistema de classificação que trata dessa comunicação. Além disso, busca-se encontrar uma ordem entre esses três períodos de quase-estabilidade do sistema de comunicação. De acordo com o modelo EEA, essa ordem pode ser de um tipo axial, na qual o período de extensão se situa em uma região intermediária entre os outros dois períodos (estabelecimento e abreviação).

Propomos, dessa forma, que a maneira como as pessoas concebem o mundo ao seu redor está diretamente relacionada à habilidade de construir categorias e elaborar sistemas de classificação, por meio dos quais diferentes estímulos (no presente caso, fotografias) podem ser tratados como equivalentes. Assim, investiga-se o entendimento que as mães têm do desenvolvimento de seus bebês na comunicação no início da vida.

As questões que se seguem fornecem um quadro relativo à nossa exploração dos diferentes aspectos relacionados à habilidade de classificar, construir categorias e conceituar que as mães apresentam em relação ao sistema de comunicação mãe-bebê aqui focalizado: 1) O que é natural e característico na concepção de mães sobre o desenvolvimento de seus bebês na comunicação no início da vida? 2) Como essa concepção é organizada em seu relacionamento com um processo constantemente em modificação, com o qual a mãe está em contínua interação? 3) Qual a estrutura subjacente a essa concepção? 4) Como essa estrutura é afetada pelo contexto sociocultural do entrevistado? 5) Essa estrutura apresenta alguma ordem?

\section{Método}

\section{Participantes}

Investigamos 48 mães que não trabalham, as quais possuíam bebês entre o sétimo e oitavo mês de vida. Metade das participantes pertencia ao nível sócio-econômico (NSE) baixo, ou classe operária $(N=24$; idade média $=23,2$; idade do bebê em meses $=27,00)$, e a outra metade ao nível sócioeconômico médio ( $N=24$; idade média $=23,9$; idade do bebê em meses=26,54).

\section{Material}

O material consistiu de 20 fotografias obtidas por meio de registros videográficos das interações mãe-bebê. Metade das fotografias representava interações FF e a outra metade interações MOB. Cada um dos subconjuntos de fotografias apresentado exibia momentos característicos do estabelecimento (três fotografias), extensão (quatro fotografias) e abreviação (três fotografias). Cada fotografia foi apresentada em um cartão de $12 \mathrm{~cm} \times 15 \mathrm{~cm}$. Utilizou-se, também, um gravador para registrar as justificativas referentes às escolhas realizadas com relação às classificações efetuadas.

\section{Procedimento}

Cada mãe foi submetida individualmente ao Procedimento de Classificações Múltiplas (PCM) e, entrevistada em duas fases (para maiores detalhes ver Roazzi, 1995). 
Na primeira fase (fase da classificação livre), foi utilizado o total de fotografias (20), sendo solicitado às mães que "formassem grupos" sem colocar nenhuma exigência quanto à maneira de processar a classificação, mais especificamente, sem nenhuma restrição quanto ao número de grupos como ao número de fotografias em cada grupo. Após cada classificação, solicitava-se que as mães justificassem as suas escolhas referentes às classificações efetuadas. Assim, de acordo com os pressupostos que norteiam uma classificação livre, pouco se requer do entrevistado; basicamente que este indique a categorização de certos elementos (fotografias) de acordo com as características que eles percebem e identificam.

$\mathrm{Na}$ segunda fase (fase da ordenação), solicitou-se aos participantes que colocassem as fotografias apresentadas em sequiência, primeiramente com o total de fotografias, em seguida com as representativas das interações FF e, por último, com aquelas referentes às interações MOB. A instrução fornecida, na segunda fase, foi a de que as mães ordenassem as fotografias em uma seqüência "de acordo com o que elas pensavam ter acontecido entre elas e os seus bebês".

\section{Resultados}

Os dados resultantes da classificação livre foram analisados por uma Multidimensional Scalogram Analysis (MSA - Lingões, 1968; Zvulun, 1978; e mais recentemente renomeada como Multidimensional Structuple Analysis - Borg \& Shye, 1995), que comparou as categorias efetuadas por cada participante para cada fotografia, sem assumir qualquer nível de similaridade sobre o significado das categorias com o mesmo número. Tendo como base a inter-relação entre os itens, o programa MSA procura colocá-los em um espaço

\section{1a. NSE Médio}

Figura 1

Projeção MSA das mães de NSE Médio (Classificação Livre)

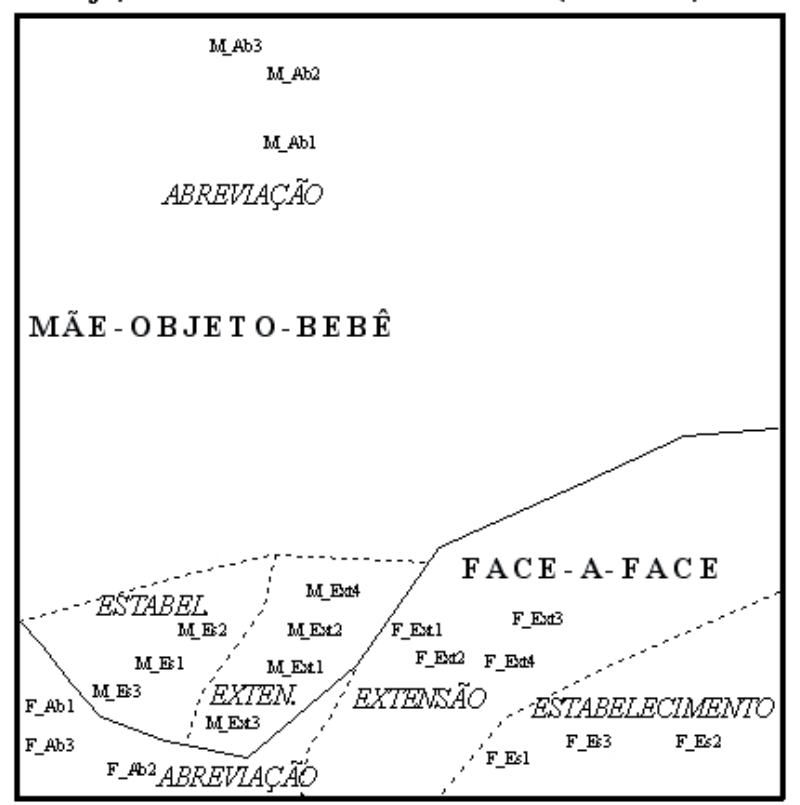

multidimensional de tal forma que os itens classificados de acordo com uma mesma categoria são representados em uma região identificável e que, portanto, o espaço possa ser subdividido em regiões que apresentem somente pontos (que correspondem ao tipo de perfil de cada item) com a mesma estrutura. Isto é, o MSA separa o espaço em regiões de tal maneira que todos os itens de um "structuple" (pertencentes a uma categoria) localizam-se em uma mesma região.

A Figura 1 apresenta os resultados MSA baseados na classificação livre realizada pelas mães de nível sócio-econômico médio (Figura 1a) e baixo (Figura 1b), respectivamente. Em ambas as figuras, o espaço é dividido em duas regiões: uma apresentando as fotografias FF e a outra, as fotografias MOB. A Figura 1b apresenta a estrutura MSA das mães de nível socioeconômico baixo, na qual cada uma das duas regiões é separada em partições axiais semelhantes. A estrutura do desenvolvimento do bebê pode ser percebida como uma ordem de três períodos, cada uma das quais ocupa uma região distinta. A ordem de fotografias é de estabelecimento, para as de extensão, para as de abreviação. A mesma ordem pode ser observada, considerando as fotografias FF, na estrutura MSA de mães de nível socioeconômico médio. Considerando a região referente às interações $\mathrm{MOB}$, as fotografias de abreviação estão localizadas na parte mais alta da projeção e os outros dois grupos de fotografias estão localizadas na parte inferior da projeção, com duas regiões muito próximas.

As Figuras 2a a 2d apresentam o SSA (Similarity Structure Analysis - Borg \& Lingões, 1987; antes denominado Smallest Space Analysis - Shye, 1985), resultados baseados na ordenação das 10 fotografias de acordo com o que as mães achavam que tinha acontecido entre elas e seus bebês. As Figuras $2 \mathrm{a}$ e $2 \mathrm{~b}$ representam a apresentação das fotografias

\section{1b. NSE Baixo}

Figura 2

Projeção MSA das mães de NSE Baixo (Classificação Livre)

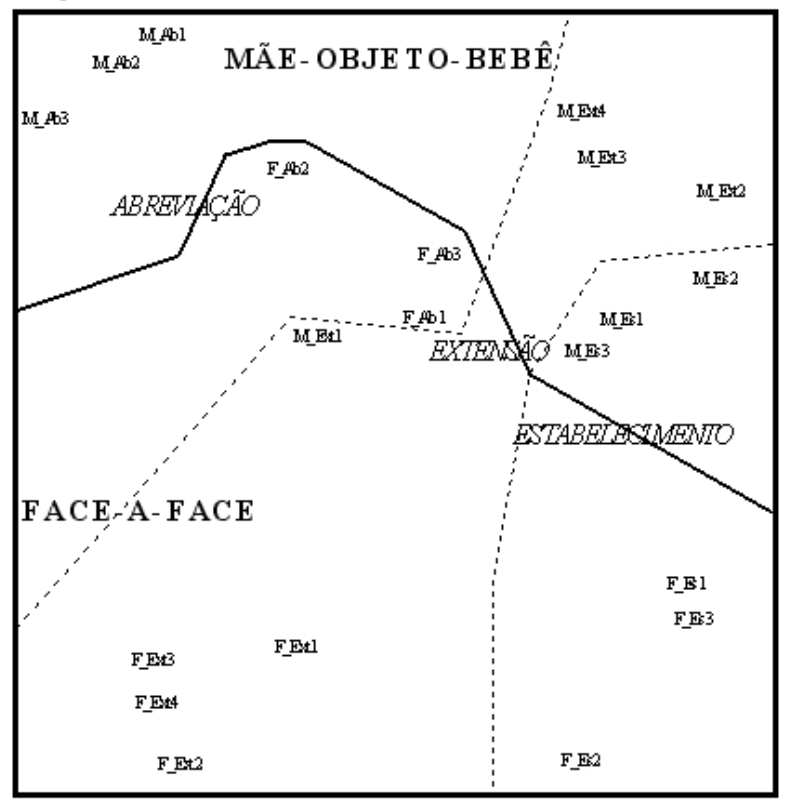

Figura 1. Projeção MAS das mães de NSE médio (1a) e de MSE baixo (1b) da classificação livre. 
FF para mães de nível socioeconômico médio e baixo, respectivamente. As Figuras $2 \mathrm{c}$ e $2 \mathrm{~d}$ representam a apresentação das fotografias MOB, para mães de nível socioeconômico médio e baixo, respectivamente. Os coeficientes de alienação das quatro figuras estão sempre bastante baixos - Figura 2a: 0,059, Figura 2b: 0,038, Figura 2c: 0,045 e Figura 2d: 0,069, indicando que a "bondade de ajustamento" entre a projeção SSA e os dados que compõem as matrizes de correlação correspondentes é adequada.

\section{2a. Face-a-Face, NSE Médio}

Figura 3

Projeção SSA das mães de NŜE Médio (Fotog. Face-a-Face)

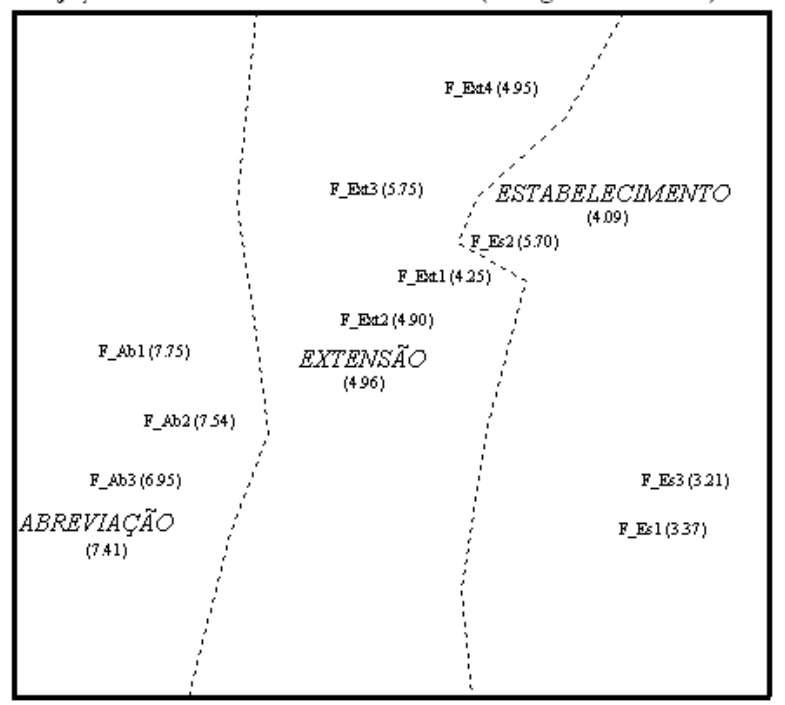

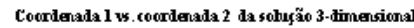

Coeficiede de Alienafäo $=0.059$

\section{2c. Mãe-Objeto-Bebê, NSE Médio}

Figura 5

Projeção SSA das mães de NSE Médio (Fotog. Mãe-Objeto-Bebê)

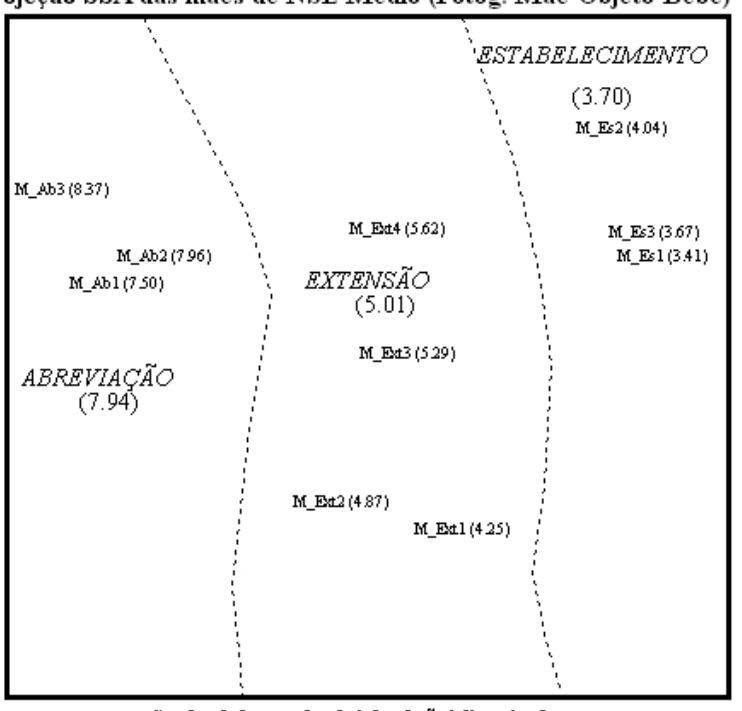

Coordenada 1 w. coordenada 2 da soluğäo 3 -finessional

Coeficiente de alienargä $=0.045$
Em todas as quatro projeções, um padrão muito similar foi obtido nos dois grupos de mães para ambos os grupos de fotografias: a estrutura do desenvolvimento do bebê pode ser percebida como uma ordem de três períodos diferentes, ocupando cada um deles uma região distinta. A ordem novamente é das fotografias de estabelecimento, para as de extensão, para as de abreviação. A média da ordenação das fotografias de abreviação é sempre a mais alta, seguida pelas fotografias de extensão e, no fim, as fotografias de

\section{2b. Face-a-Face, NSE Baixo}

Figura 4

Projeção SSA das mães de NSE Baixo (Fotog. Face-a-Face)

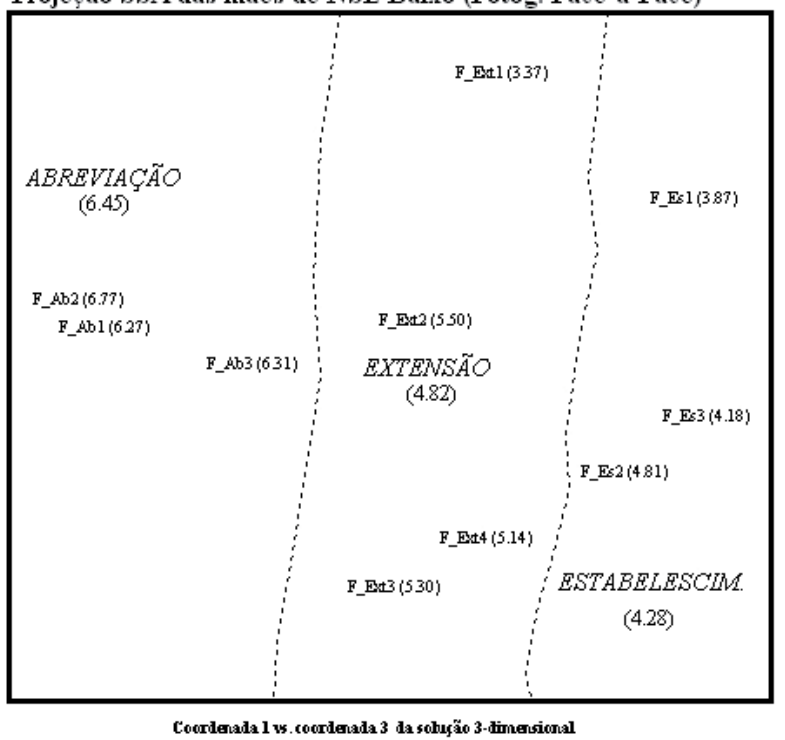

Coeficiente de atienarã $0=0038$

2d. Mãe-Objeto-Bebê, NSE Baixo

Figura 6

Projeção SSA das mães de NSẼ Baixo (Fotog. Mãe-Objeto-Bebê)

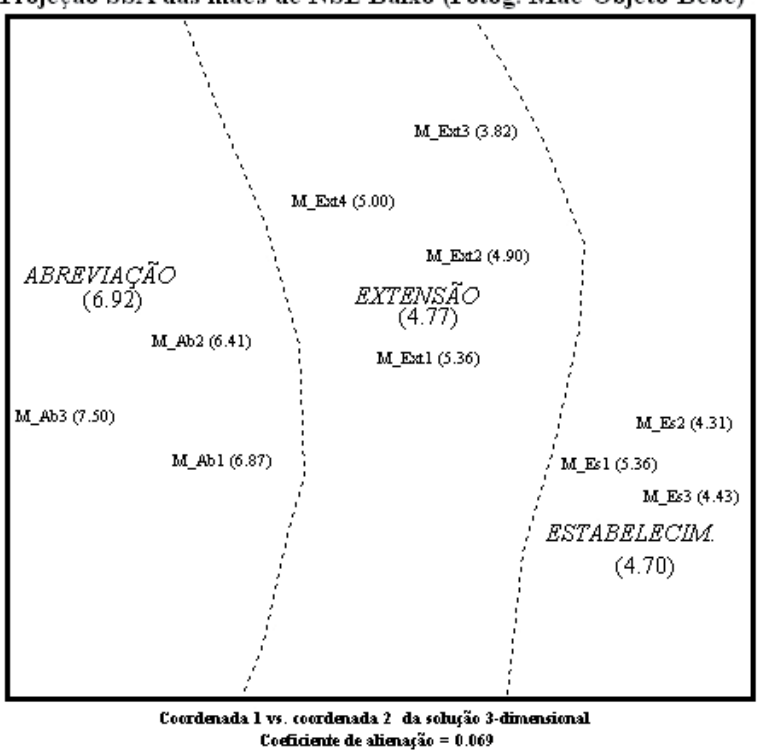

Figura 2. Projeções SSA das fotos face-a-face (2a - mães de NSE médio, 2b - mães de NSE baixo) e das fotos mãe-objeto-bebê (2c - mães de NSE médio, 2d - mães de NSE baixo). 
3a. NSE médio e baixo

Figura 7

Projeção SSA das mães de NSE Médio e Baixo (Todas as Fotog.)

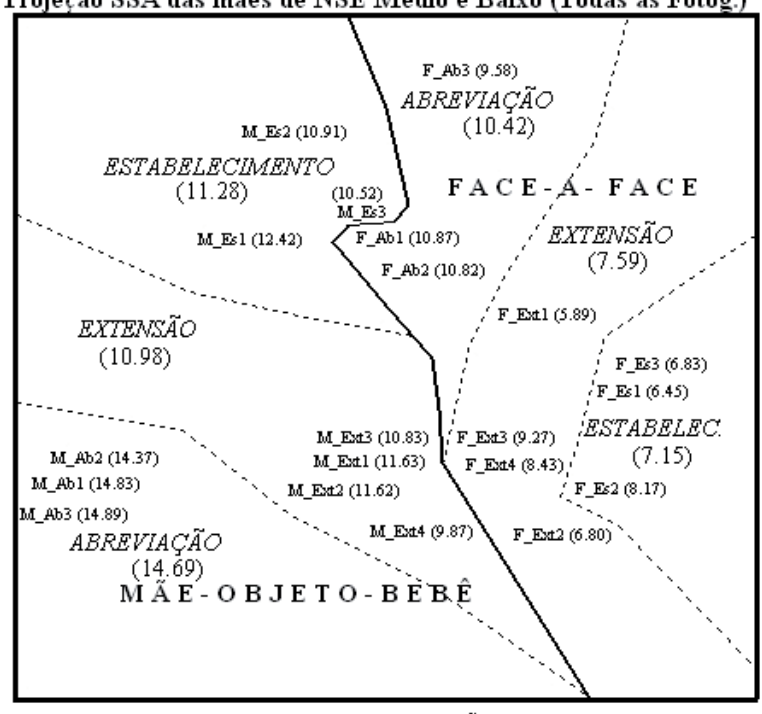

Coordenada 1 vs. coordenada 3 da solução 3-dimensional

Cofficiente de alienară $=0.082$

3c. NSE baixo

Figura 9

Projeção SSA das mães de NSE Baixo (Todas as Fotog.)

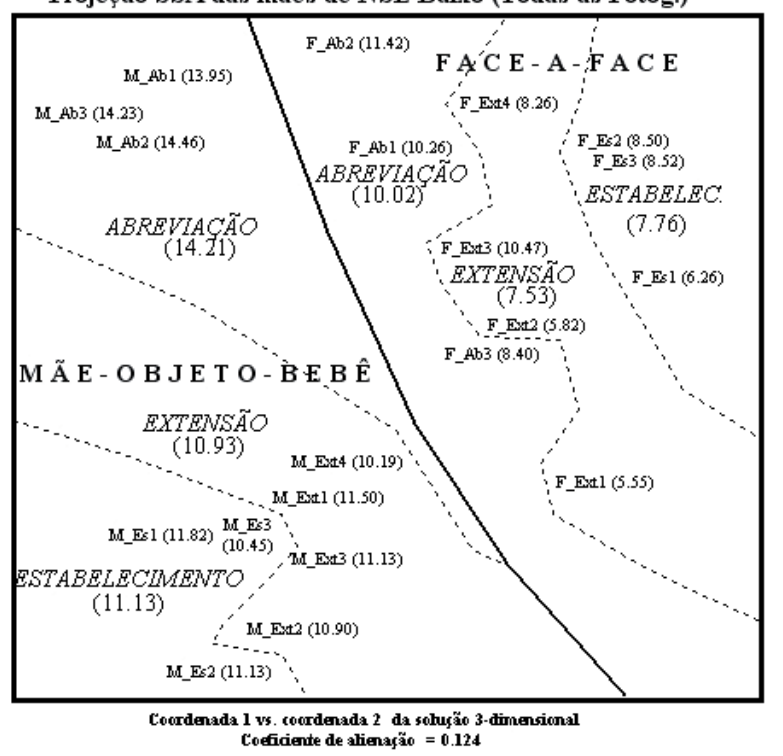

Figura 3. Projeções SSA de todas fotos considerando as mães de NSE médio e baixo (3a), de NSE médio (3b) e de NSE baixo (3c)

estabelecimento. Comparando a média das fotografias de extensão - localizadas na região central da estrutura axial -, a diferença era sempre maior em relação às fotografias de abreviação (Figura 2a: 2,45, Figura 2b: 1,03: Figura 2c: 2,93; Figura 2d: 2,15) do que em relação às fotografias de estabelecimento (Figura 2a: 0,87, Figura 2b: 0,34, Figura 2c: 3b. NSE médio

Figura 8

Projeção SSA das mães de NSE Médio (Todas as Fotog.)

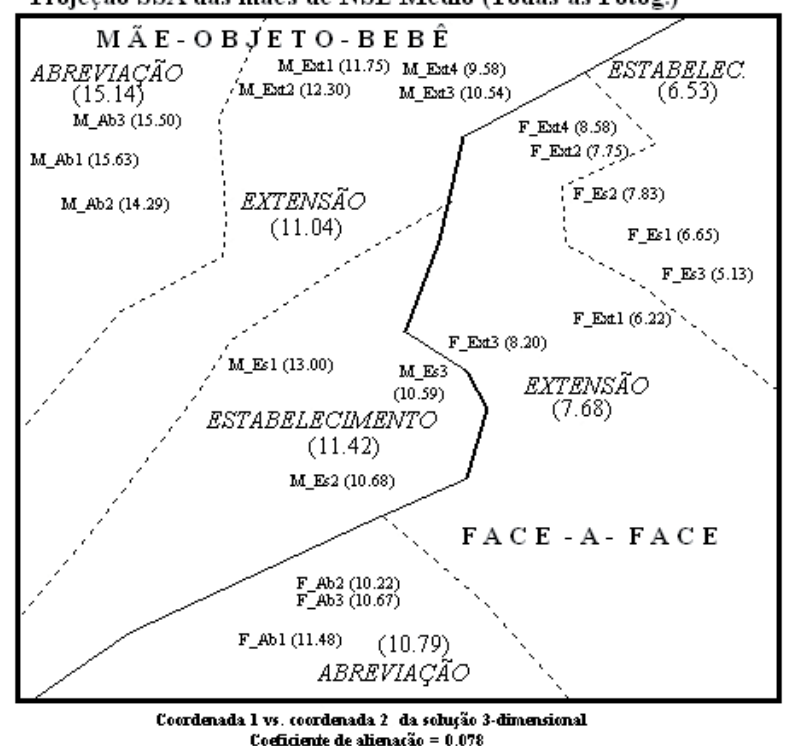

1,31, Figura 2d: 0,07). Isso significa que períodos de extensão são vistos como mais próximos aos períodos de estabelecimento do que aos períodos de abreviação. Essa observação está em concordância com o que foi observado nas estruturas MSA baseadas na classificação livre, nas quais fotografias de extensão e estabelecimento estão sempre mais próximas umas das outras do que elas estão para o terceiro grupo de fotografias que representam períodos de abreviação da comunicação.

As Figuras 3a a $3 \mathrm{c}$ apresentam os resultados SSA baseados na ordenação das 20 fotografias, por todas as mães. Os coeficientes de alienação das três figuras são sempre baixos - Figura 3a: 0,082, Figura 3b: 0,078, Figura 3c: 0,124, indicando que a "bondade de ajustamento" entre a projeção SSA e os dados que compõem as matrizes de correlação correspondentes é adequada. Em todas as três projeções o espaço é claramente dividido em duas regiões contíguas correspondendo aos dois tipos de comunicação, característicos da faixa etária investigada: uma representando as fotografias FF e outras fotografias MOB. Cada uma dessas duas regiões nas três figuras são também divididas em três regiões, correspondendo aos três conceitos, EEA, propostos por Lyra e colaboradores (Fogel \& Lyra, 1997; Lyra, 2000, 2006a, Lyra, 2006b, Lyra, 2007, Lyra, no prelo; Lyra \& Souza, 2003; Lyra \& Winegar, 1997) como períodos de quase-estabilidade do sistema de comunicação mãe-bebê no início da vida. Mais especificamente, em todas as três projeções, um padrão similar foi obtido nas duas regiões FF e MOB: a estrutura do desenvolvimento do bebê pode ser percebida como uma ordem de três diferentes períodos, cada um dos quais ocupando uma região distinta. A ordem novamente - como nas figuras apresentadas acima - é das fotografias de estabelecimento, para as de extensão, para as de abreviação.

Nas Figuras 3a e 3b, a média da ordenação das fotografias de abreviação é sempre mais alta, seguidas pelas fotografias de extensão e, no fim, as fotografias de estabelecimento. Comparando a média da ordenação das fotografias de ex- 
tensão - localizadas na região central da estrutura axial -, a diferença foi sempre maior com as fotografias de abreviação (Figura 3a: FF 2,83 e MOB 3,71; Figura 3b: FF 3,71 e MOB $4,10)$ que com as fotografias de estabelecimento (Figura $3 \mathrm{a}$ : FF 0,44 e MOB 0,30; Figura 3b: FF 1,15 e MOB 0,63). Isso mostra que os períodos de extensão são percebidos muito mais próximos aos períodos de estabelecimento que aos períodos de abreviação. Nessas duas Figuras ( $3 a$ e $3 b$ ), é também possível ver que, nas regiões de FF, 1) a média da ordenação mais baixa começa com as fotografias de estabelecimento, seguidas pelas fotografias de extensão e pelas fotografias de abreviação (para Figura $3 \mathrm{a}$ as médias são 7.15, 7.59 e 10.42, e para Figura 3b a médias são 6,53, 7,68 e 10,79, respectivamente); 2) nas regiões de MOB (Figura $3 b$ ), a média da ordenação mais baixa começa com fotografias de extensão, seguidas pelas fotografias de estabelecimento e pelas fotografias de abreviação (para Figura 3a as médias são 10,98, 11,28 e 14,69, e para Figura 3b as médias são 11,04, 11,42 e 15,42, respectivamente). Essa inversão da média da ordenação de fotografias de extensão seguidas por fotografias de estabelecimento (ao invés de fotografias de estabelecimento seguidas por fotografias de extensão), na região MOB, também é observada na Figura 3c, que apresenta a solução SSA de mães de classe operária, não apenas na região $\mathrm{MOB}$, mas também na região $\mathrm{FF}$. No entanto, é importante mostrar que essa diferença é bastante pequena quando comparada às diferenças entre extensão e abreviação (ver diferenças apresentadas no parágrafo anterior).

\section{Discussão}

Comparamos duas abordagens teórico-metodológicas distintas e investigamos a concepção que mães apresentam acerca do desenvolvimento da comunicação mãe-bebê, considerando três conceitos que descrevem padrões de organização desenvolvimentais desse sistema de comunicação, denominados estabelecimento, extensão e abreviação. Esses conceitos integram o modelo EEA e estão baseados na perspectiva teórico-metodológica dos Sistemas Dinâmicos. Eles foram propostos a partir da observação longitudinal e análise microgenética do estudo de casos, investigando as trocas mãe-bebê ao longo dos oito primeiros meses de vida pós-natal do bebê.

Dois aspectos principais merecem ser enfatizados. Os três conceitos (EEA), padrões de organização característicos do desenvolvimento desse sistema de comunicação, são períodos de quase-estabilidade atingidos pelas interações mãe-bebê (tanto das trocas FF como daquelas MOB). A perspectiva dos Sistemas Dinâmicos, que dá suporte para esta descrição conceitual do desenvolvimento, propõe que todo desenvolvimento é um processo contínuo (em transformação constante, portanto) que compreende períodos de maior estabilidade e períodos de menor estabilidade (Fogel, Lyra \& Valsiner, 1997). Assim, esses conceitos referem-se a períodos que descrevem um equilíbrio dinâmico que caracteriza o desenvolvimento do sistema de comunicação estudado. Outra tendência fundamental da perspectiva dos Sistemas Dinâmicos (Thelen \& Ulrich, 1991; Lyra, 2000) está na utilização de uma análise microgenética das pequenas transformações das trocas entre os parceiros, mãe e bebê, identificadas e analisadas observando-se o tempo real (segundo a segundo, Thelen \& Ulrich, 1991).
Trata-se, portanto, de conceitos essencialmente processuais, que visam a compreender o desenvolvimento da comunicação como um processo em constante transformação embora eles representem períodos de quase-estabilidade, passíveis de serem identificados de forma macroscópica. Investigamos, neste trabalho, a capacidade das mães de conceituar o desenvolvimento da comunicação mãe-bebê, tendo como base esses três conceitos, EEA. Em outras palavras, se esses períodos correspondem a mudanças no desenvolvimento desse sistema de comunicação, observáveis a um nível macroscópico, então os conceitos de EEA podem ser identificados nas habilidades das mães ao construírem um sistema de classificação para esse desenvolvimento. Essa hipótese foi confirmada tanto em relação às trocas FF como àquelas $\mathrm{MOB}$.

Os resultados, tanto da apresentação livre como da apresentação dirigida, para ambos os grupos de mães, revelaram uma conceituação do desenvolvimento da comunicação mãebebê que diferencia os três períodos - EEA - desse sistema de comunicação. Existe, também, uma ordem gradativa, a extensão localiza-se no meio, entre o estabelecimento e a abreviação. $\mathrm{O}$ aspecto dicotômico relativo aos dois tipos de trocas, FF e MOB, pode, também, ser claramente dividido em duas partições contíguas, correspondendo a esses dois tipos de trocas. Assim, encontramos, na concepção que as mães têm sobre o desenvolvimento da comunicação mãe-bebê no início da vida pós-natal, claramente, suporte para o que propõe o modelo EEA, ou seja, essa teoria dos três conceitos. O estabelecimento, a extensão e a abreviação correspondem, nessa sequiência, às idéias que as mães formam acerca do processo de desenvolvimento da comunicação mãe-bebê durante os oito primeiros meses de idade do bebê.

Esses resultados são particularmente interessantes, porque demonstram semelhança na conceituação das mães tanto em relação ao desenvolvimento das trocas FF como das trocas MOB. Todavia, se existe alguma discrepância nessa ordem, ou seja, primeiro o estabelecimento depois a extensão e, finalmente, a abreviação, esse fato se dá em relação às trocas mediadas pelo objeto. Foi na conceituação das trocas MOB que houve uma menor diferenciação (valores das médias) entre o estabelecimento e a extensão, embora a abreviação apareça bem distinta para mães de ambas as classes sociais estudadas. Se realmente essa menor diferenciação diz de uma tendência conceitual das mães, pode-se supor que o domínio da comunicação criada, predominantemente na base das emoções, característico das trocas FF (Bråten, 1988; Trevarthen, 1998), desperta nas mães um grau de acuracia maior que aquele que advém de uma comunicação mais "evoluída", característica das trocas mediadas pelo objeto (MOB). Essas últimas estão baseadas em capacidades cognitivas mais desenvolvidas do bebê, representada, por exemplo, pela emergência da noção de intencionalidade do parceiro (Bråten, 1988; Trevarthen, 1998).

Finalizando, ressaltamos que este estudo confirma e amplia o poder da teoria dos três conceitos - estabelecimento, extensão e abreviação - que integra o modelo EEA proposto por Lyra e colaboradores para o estudo e compreensão do processo de desenvolvimento da comunicação mãe-bebê durante os primeiros meses de vida pós-natal do bebê (Fogel \& Lyra, 1997; Lyra, 2000, 2006a, Lyra, 2006b, Lyra, 2007, Lyra, no prelo; Lyra \& Souza, 2003; Lyra \& Winegar, 1997). 


\section{Referências}

Bilsky, W. (2003). A teoria das facetas: noções básicas. Estudos de Psicologia, 8(3), 357-365.

Borg, I. \& Lingões, J.C. (1987). Multidimensional similarity structure analysis. New York: Springer.

Borg, I. \& Shye, S. (1995). Facet theory form and content. Newbury Park: Sage.

Bråten, S. (1988). Dialogic mind: the infant and the adult in proconversation. Em M. Carvalho (Org.), Nature, cognition and system (pp. 187-205). Dordrecht: Kluwer Academic Press.

Canter, D. (Org.) (1985). Facet theory: Approaches to social research. New York: Springer-Verlag.

Coleman, P. \& Watson, A. (2000). Infant attachment as a dynamic system. Human Development, 43, 295-313.

Fogel, A. \& Lyra, M. C. D. P. (1997). Dynamics of development in relationships. Em F. Masterpasqua \& P. Perna (Orgs.), The psychological meaning of chaos: Translating theory into practice (pp. 75-94). Washington: American Psychological Association.

Fogel, A. (1993). Developing through relationships. Origins of communication, self and culture. Chicago: University of Chicago Press.

Fogel, A., Lyra, M. C. D. P. \& Valsiner, J. (1997). Perspectives on indeterminism and development. Em A. Fogel, M. C. D. P. Lyra, \& J. Valsiner (Orgs.), Dynamics and indeterminism in developmental and social processes (pp. 1-10). Mahwah: Lawrence Erlbaum.

Granott, N. \& Parziale, J. (2002). Microdevelopment: A processoriented perspective for studying development and learning. Em N. Granott, \& J. Parziale, (Orgs.), Microdevelopment. Transition processes in development and learning (pp. 1-28). Cambridge: Cambridge University Press.

Guttman, L. (1991). Louis Guttman: In memoriam - Chapters from an unfinished textbook on facet theory. Jerusalém: The Israel Academy of Sciences and Humanities.

Lewis, M. D. (1995). Cognition-emotion feedback and selforganization of developmental paths. Human Development, 38, 71-102.

Lewis, M. D. (2000). The promising of dynamic systems approaches for an integrative account of human development. Child Development, 71(1), 36-43.

Lingões, J. C. (1968). The multivariate analysis of qualitative data. Multivariate Behavioral Research, 3, 61-94.

Lyra, M. C. D. P. (2000). Desenvolvimento como processo de mudança em um sistema de relações historicamente construído: contribuições do estudo da comunicação no início da vida. Psicologia: Reflexão e Crítica, 13(2), 257-268.

Lyra, M. C. D. P. (2006a). Desenvolvimento como processo de mudança. Em A. G. Spinillo \& L. L. Meira (Orgs.), Psicologia cognitiva: cultura, desenvolvimento e aprendizagem. Recife: Editora da Universidade Federal de Pernambuco.

Lyra, M. C. D. P. (2006b). O Modelo EEA para a investigação da emergência e desenvolvimento da comunicação e do self: bases conceituais e fundamentos teórico-metodológicos. Estudos de Psicologia 11(1), 25-33.

Lyra, M. C. D. P. (2007). O Modelo EEA: definições, unidade de análise e possíveis aplicações. Psicologia: Reflexão e Crítica, 20(1), 87-95.

Lyra, M. C. D. P. (no prelo). Mother-infant communication development and the emergence of self: The contributions of dynamic systems and dialogism. Em C. Lightfoot \& M. C. D. P. Lyra (Orgs.), Challenges and strategies for studying human development in cultural contexts. Roma: Carlo Amore Edizioni.

Lyra, M. C. D. P. \& Souza, M. (2003). Dynamics of dialogue and emergence of self in early communication. Em Josephs, I. (Org.), Dialogicality in development. Vol. 5. Advances in Child Development Culturally Structured Environments (pp. 51-68). Westport: Praeger Publishers.

Lyra, M. C. D. P. \& Winegar, L. T. (1997). Processual dynamics of interactions through time: Adult-child interactions and process of development. Em A. Fogel, M. C. D. P. Lyra \& J. Valsiner (Orgs.), Dynamics and indeterminism in developmental and social processes (pp. 93-109). Mahwah: Lawrence Erlbaum.

Masterpasqua, F. \& Perna, P. (Orgs.) (1997). The psychological meaning of chaos: Translating theory into practice. Washington: American Psychological Association.

Molenaar, P. C. M. \& Valsiner, L. (2005). How generalization works through the single case: A simple idiographic process analysis of an individual psychotherapy. International Journal of Idiographic Science, 1. Retirado em 25/04/2006 de http://www.valsiner.com

Pantoja, A. P. F. \& Nelson-Goens, G. C. (2000). Desenvolvimento da vida emocional durante o segundo ano de vida: narrativas e sistemas dinâmicos. Psicologia: Reflexão e Crítica, 13(2), 269-280.

Pedrosa, M. I., Carvalho, A. M. A. \& Império-Hamburger, A. (1997). From disordered to ordered movement: Attractor configuration and development. Em A. Fogel, M. C. D. P. Lyra \& J. Valsiner (Orgs.), Dynamics and indeterminism in developmental and social processes (pp. 135-151). Mahwah: Lawrence Erlbaum.

Roazzi, A. (1995). Categorização, formação de conceitos e processos de construção de mundo: Procedimento de classificações múltiplas para o estudo de sistemas conceituais e sua forma de análise através de métodos de análise multidimensionais. Cadernos de Psicologia, 1, 1-27.

Roazzi, A., Wilson, M. \& Federicci, F. C. B. (2001). A estrutura primitiva da representação social do medo. Psicologia: Reflexão e Crítica, 14(1), 57-72.

Roazzi, A. \& Dias, M. G. B. B. (2001). Teoria das facetas e avaliação na pesquisa social transcultural: Explorações no estudo do juízo moral. Em Conselho Regional de Psicologia - 13a Região PB/RN (Org.), A diversidade da avaliação psicológica: Considerações teóricas e práticas (pp. 157-190). João Pessoa: Idéia.

Sherif, M. \& Sherif, C. (1969). Social psychology. New York: Harper \& Row.

Silva, M. S., Lyra, M. C. D. P., \& Roazzi, A. (2001). The Development of Mother-Object-Infant Communication: Methodological contributions from Facet Theory. Em D. Elizur (Org.), Facet theory: Integrating theory construction with data analysis (pp. 367-383). Prague: Matfyzpress.

Shye, S. (1985). Smallest space analysis (SSA). International encyclopedia of education. Oxford: Pergamon.

Thelen, E. \& Ulrich, B. D. (1991). Hidden skills: A dynamic systems analysis of treadmill stepping during the the first year. Monographs of the Society for Research in Child Development, 56, (1).

Thelen, E. \& Smith, L. B. (1994). A dynamic system approach to the development of cognition and action. Cambridge: The MIT Press.

Trevarthen, C. (1998). The concept and foundations of infant intersubjectivity. Em S. Bråten, (1998) (Org.), Intersubjective 
communication and emotion in early ontogeny (pp. 15-46). Cambridge: Cambridge Univerty Press.

Valsiner, J. (2002). Irreversibility of time and ontopotentiality of signs. Estudios de Psicologia, 23(1), 49-59.

van Geert, P. (2002). Developmental dynamics, intention actions and fuzzy sets. Em N. Granott, \& J. Parziale, (Orgs.), Microdevelopment. Transition processes in development and learning Sets (pp. 319343). Cambridge: Cambridge University Press.

van Geert, P. (2003). Dynamic systems approaches and modeling of developmental processes. Em J. Valsiner \& K. Connolly (Orgs.), Handbook of developmental psychology, (pp. 640-672). Londres: Sage.
Vygotsky, L. S. (1962). Thought and language. Cambridge: MIT Press. (Trabalho original publicado em 1934)

Zvulun, E. (1978). Multidimensional Scalogram Analysis: the method and its applications. Em S. Shye (Org.), Theory construction and data analysis in the behavioural sciences (pp-237-255). London: Jossey-Bass Publishers.

Recebido em 12.07.2006

Primeira decisão editorial em 26.07.2007

Versão final em 11.10.2007

Aceito em 11.10.2007 